\title{
ERRATUM
}

\section{Maximizing Lifetime for Data Aggregation in Wireless Sensor Networks}

\author{
Yuan Xue • Yi Cui • Klara Nahrstedt
}

Published online: 16 June 2006

(C) Springer Science + Business Media, LLC 2006

\section{Mobile Networks and Applications (2006) 10(6) 853-864 \\ DOI 10.1007/s11036-005-4443-7}

Author Klara Nahstedt's affiliation was listed incorrectly. The correct affiliation is:

Department of Computer Science

University of Illinois at Urbana-Champaign Urbana, IL 61801, U.S.A.

The online version of the original can be found at: http:// dx.doi.org/10.1007/s11036-005-4443-7

Yuan Xue

Department of Electrical Engineering and Computer Science,

Vanderbilt University, VU Station B 351824,

Nashville, TN 37235, USA

Yi Cui

Department of Electrical Engineering and Computer Science, Vanderbilt University, VU Station B 351824,

Nashville, TN 37235, USA

Klara Nahrstedt

Department of Computer Science,

University of Illinois at Urbana-Champaign,

Urbana, IL 61801, USA 